\title{
Small Traveling Salesman Problems
}

\author{
Richard H. Warren \\ Retired: Lockheed Martin Corporation, King of Prussia, PA 19406, USA \\ Address: 403 Bluebird Crossing, Glen Mills, PA 19342-3362, USA \\ Email: rhw3@psu.edu
}

\begin{abstract}
This paper illustrates fundamental principles about small traveling salesman problems (TSPs) which are a current application for quantum annealing computers. The 2048 qubit, quantum annealing computer manufactured by D-Wave Systems is estimated to be able to solve all TSPs on 8 cities, which advances a recent 4-city result on a quantum simulator. Additionally, the D-Wave quantum computer is expected to find all optimal tours for each TSP. To prepare for this, we show the expected quantum output for 5,000 randomly generated TSPs on 6,8 and 10 cities. The examples in the TSPLIB have 14 or more cities. These are too large for the current quantum annealing processors. We have included an annotated bibliography about solving the TSP on a quantum computer.
\end{abstract}

Keywords: Traveling salesman, optimal tour, combinatorial analysis, discrete optimization, quantum annealing

AMS Classification Codes: 68R05, 81P68, 90C27

\section{Introduction}

Recently first steps have been taken to solve the traveling salesman problem (TSP) using the computational advantages promised by quantum algorithms [13]. As with any emerging effort, the initial steps have been limited and focused on a small number of cities that fit on the hardware.

The major contributions of this paper are for 6,8 and 10 city TSPs. We present empirical evidence based on 15,000 random samples, 5,000 for each number of cities. The maiority of evidence indicates that the number of optimal tours and the length of an optimal tour increase together. All of the data shows that as the number of cities increases, the number of optimal tours increases. In all cases, the distances between cities are random integers in the interval [1,21].

Outside the quantum world, the traveling salesman problem continues to be a hot topic that attracts wide interest. The problem is easy to explain and has several diverse applications [9, 18], as well as theoretical studies $[2,20]$. There are many fascinating ways to solve it, usually approximately. Given a set of cities and the directed distances between each pair of cities, the TSP asks for a shortest route that visits each city exactly once and returns to the starting city. There are excellent, non-quantum TSP surveys $[5,7,10]$.

Quantum computing, particularly quantum annealing [3], is a new paradigm for discrete optimization that could use TSP benchmarks for the hardware. Quantum computing opens the possibility of large speedup with high probability of optimal answers, but requires new techniques for solving the TSP [11, 19].

The paper is organized as follows: Section 2 has references for other reports that use quantum computing algorithms for solving the TSP. Section 3 contains the results from a study of the expected output for TSPs from current quantum annealing computers. The output is envisioned to be all optimal tours, since these are obtained on a quantum annealing simulator. We have classified this output for 6,8 and 10-city TSPs which is the estimated size that can be solved currently. The last section points to future quantum work for the TSP.

Our notation: Let the cities be designated $1,2, \ldots, n$. Let $d_{i j}$ be the distance from city $i$ to city $j$. We do not assume that $d_{i j}=d_{j i}$, unless a symmetric TSP is indicated. The $n$ by $n$ matrix $\left[d_{i j}\right]$ is called the 
distance matrix for the TSP. A tour is a cyclic permutation of the cities, i.e., a permutation that has one cycle. The TSP asks for a tour of the cities that returns to the starting city and minimizes the distance traveled, called an optimal tour. The number of tours for the $n$-city TSP is $(n-1)$ !. A subtour is a cyclic permutation of a proper subset of the cities.

\section{Literature Relating the Traveling Salesman Problem to Quantum Computing}

In this section we describe the content of published work that connects the TSP to quantum computing. In some cases we comment about issues.

References $[6,8,11,12,15,19]$ formulate techniques to adjust the TSP to a quantum computer and provide an algorithm to solve the TSP. A major issue is to avoid an exponential number of constraint equations, since that number of relations may frustrate the quantum process and certainly will impede the input. Constraints are needed to block subtours. These are circular routes through a subset of cities which does not contain all of the given cities.

Dahl[6] has an outstanding TSP presentation based on lengths of potential solutions that are constrained so that each city is visited once and subtours do not occur. Using Python code, Dahl feeds his objective function and constraints into a D-Wave compiler, called BlackBox, which interfaces with the quantum hardware. Python code is included in [6]. Output from the quantum machine is shown and interpreted. Apparently, the BlackBox compiler is no longer supported by D-Wave and has been replaced by other compilers.

Inoue $[8]$ introduces an objective function in his formula (4) that contains two products. It has been shown $\lceil 11,19]$ that one product suffices. Inoue has three constraints that block undesired results. He does not mention baring subtours, but this appears to happen by the ordering criteria in his subscript $i$ on $t_{i a}$. The work to obtain a cost Hamiltonian, designated (5) in [8], is greatly obscured, hampered by a new term $\delta$. and contains an apparent constant $C$ that is not in the Ising model (3) in [8].

Lucas [11] has an excellent description of quantum annealing from the perspective of a physicist. Lucas formulates the Hamiltonian cycles problem for D-Wave quantum computing and adapts it to the TSP. His methods to attain an optimal solution for the TSP parallel the techniques in [19]. No implementations are reported in [11]. The main innovation, as in [19], is to use the order of visiting cities as a subscript on the variables for the problem.

Martoňák, Santoro and Tosatti $\lceil 12\rceil$ authored one of the original papers about solving the TSP by quantum annealing. It was published several vears before D-Wave Systems announced a pursuit of quantum annealing for commercial processors. The authors use the 1986 paper by Hopfield and Tank as a basis for their TSP quantum algorithm, and use 2-opt moves for improvement. When tested on the printed circuit board instance pr1002 of the TSPLIB [17], simulated quantum annealing converged faster than standard thermal simulated annealing.

Santoro and Tosatti $\lceil 15\rceil$ continue the work in $\lceil 12\rceil$. They present an excellent overview of the physical concepts for quantum annealing, an adaptation of this theory to an algorithm for the TSP, and benchmark results of their algorithm for the printed circuit board instance pr1002 of the TSPLIB [17]. It is a structured, symmetric TSP with 1002 cities whose optimal tours have length 259045. For pr1002 the quantum TSP algorithm was executed in a simulated quantum annealing environment that outperformed standard thermal simulated annealing in both time and accuracy.

Warren [19] uses the 1986 paper by Hopfield and Tank as a basis for a TSP quantum algorithm that has the order of visiting cities as a subscript on the variables for the problem. This innovation captures the subtour constraint in the subscripts. Later it is used in [11]. The TSP results in [19] can be improved by capitalizing on the $n$-fold degeneracy of the initial city for a tour on the $n$-city problem, validating the implementation on a D-Wave processor, and using the algorithm in [19] to solve random TSPs on a D-Wave processor. The equivalence between the adiabatic quantum model and the quantum circuit model that is mentioned in [19] does not apply to quantum annealing as implemented by D-Wave.

The quantum annealing function for the TSP presented by Suzuki et al. [16 pages 227-228] is flawed because it lacks a constraint to prevent subtours. It is not known how to constrain subtours efficiently for a D-Wave processor when the subscripts on the binary variable are pairs of cities, as in [16]. A fully 
automated, efficient method to constrain subtours for the asymmetric TSP is a major hurdle for digital computing $\lceil 10\rceil$.

Moser [14] suggests a quantum hardware design for the TSP and illustrates the effect for a 6-city TSP. It appears that the proposed hardware is neither circuit theory nor quantum annealing.

Chen and his coauthors $[4]$ use the notation and formulation of [12] for a quantum solution to a 4-city TSP on a nuclear magnetic resonance quantum simulator.

Bang and his colleagues $\lceil 1\rceil$ report a quadratic speedup for a quantum heuristic TSP algorithm that generalizes the structure of Grover search for the Gaussian distribution, as compared to the classical heuristic algorithm.

In summary, papers $\lceil 11,19\rceil$ use the same method and score highest on technical content. Article [6] is outstanding, but apparently has lost its usefulness due to the demise of the BlackBox interface. The technical presentation in $\lceil 8\rceil$ is partially unclear. Papers $\lceil 1,12,15\rceil$ use a heuristic algorithm that may not find an optimal result. Papers $[4,14]$ demonstrate their efforts on small TSPs with 4 and 6 cities, respectively.

\section{$3 \quad$ Simulating the Quantum Annealing Solution of TSPs}

In this section we discuss expected results of solving TSPs on a D-Wave Systems quantum annealing computer. The current quantum chip has 2048 quantum bits ${ }^{1}$, called qubits, that can be annealed to represent a minimum for a discrete optimization problem. The inputs are biases on the qubits and on the connections between qubits. The small number of qubits and sparse connections on this machine limit TSPs, as quantum formulated in $[11,19]$, to about 8 cities.

Programming a quantum annealing computer means giving it one instruction so that when the machine is super cooled and reaches a minimum energy, each qubit can be interpreted as a 0 or 1 representing a minimum solution. The problem for the quantum computer to solve can be regarded as a landscape where we are looking for a minimum value. The annealing process can be described as a cloud settled on the landscape that is able to detect a global minimum. In a sense all points on the landscape are examined simultaneously to determine a minimum. Essentially each result is sampled from a Boltzmann distribution. Therefore it is repeated at least 100 times, often 1000 times, to ensure a minimum is found. As a result, several points that have the same global minimum may be identified. In terms of the TSP, this means that several optimal tours may be identified. This has led us to study the distribution of optimal tours for randomly generated TSPs with 6,8 and 10 cities.

The range of coefficients for the current D-Wave quantum simulator is values in the interval [-10, 10]. Integer values are preferred to ensure adequate energy distinctions. Therefore, the distances between cities in the study were integer values in the range $[1,21]$ that were randomly generated using clock time as the seed. The transformation $\mathrm{x}->(\mathrm{x}-11)$ maps distances in $[1,21]$ to values in $[-10,10]$ and preserves ordering of tours according to their length.

We randomly generated the distances between cities for 1000 TSPs, determined all of the optimal tours for each TSP, averaged the length of the optimal tours per number of optimal tours, and plotted the results. This was done 5 times, which we call series. Figures $1-3$ show these results as line graphs for 6,8 and 10 city TSPs.

More than $99 \%$ of the 15,000 TSPs for 6,8 and 10 cities had only 1, 2 or 3 optimal tours. The data points in Figures 1 - 3 for 1, 2 or 3 optimal tours are very close, and in all cases except two, the line graphs between these data points show positive slope. The latter indicates that as the average length of an optimal tour increases, the number of optimal tours increases, which is a valid statistical implication for large samples.

Figure 4 compares the results for 6,8 and 10 cities. The trend of the data for 2 and 3 optimal tours continues for 4 - 7 optimal tours, but is obscure in Figure 4. Therefore, we show the data in Table 1.

1 The chip is a $16 \times 16$ two-dimensional array of blocks. Each block holds 8 qubits connected as a primal graph that D-Wave calls Chimera. There is limited connectivity between qubits of adjacent blocks. Please see [3 Figure 1] for an $8 \times 8$ array and [3 Figure 2] for the connectivity between adjacent blocks. 


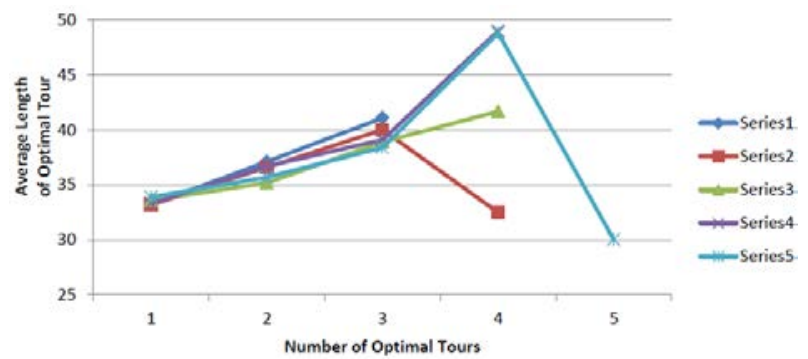

Figure 1. 6 cities - 1000 TSPs per series (color online)

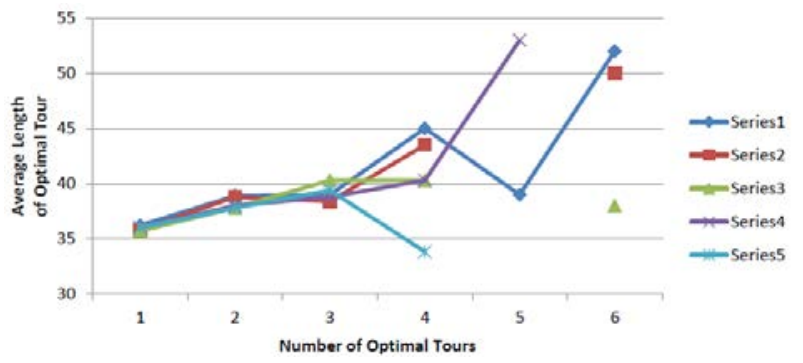

Figure 2. 8 cities - 1000 TSPs per series (color online)

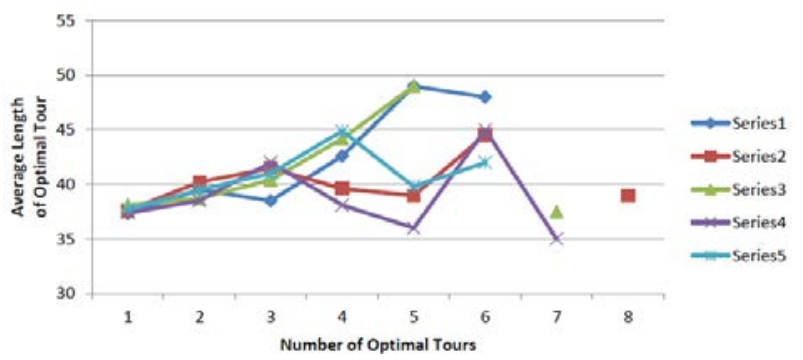

Figure 3. 10 cities - 1000 TSPs per series (color online)

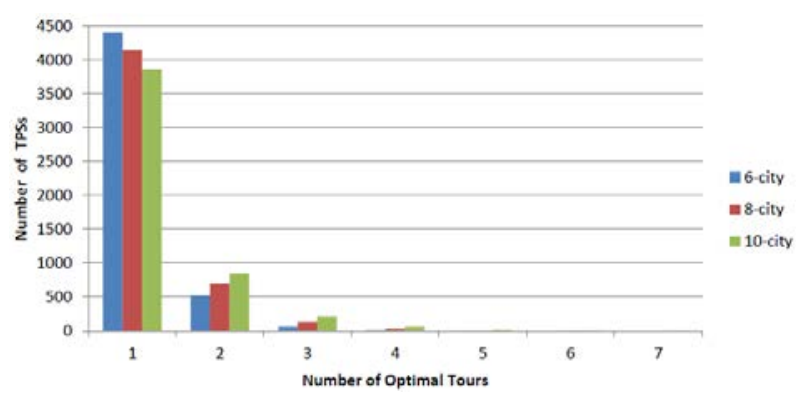

Figure 4. Distribution of 5,000 random TSPs for 6, 8, 10 cities (color online) 
Table 1. Distribution of random TSPs for 6,8 and 10 cities.

\begin{tabular}{llll}
\hline & 6 Cities & 8 Cities & 10 Cities \\
\hline 2 Optimal Tours & 521 & 696 & 844 \\
3 Optimal Tours & 61 & 131 & 209 \\
4 Optimal Tours & 11 & 30 & 60 \\
5 Optimal Tours & 1 & 3 & 15 \\
6 Optimal Tours & 0 & 3 & 8 \\
7 Optimal Tours & 0 & 0 & 3 \\
\hline
\end{tabular}

The weighted average number of optimal tours for 6-city TSPs was 1.1, for 8-city TSPs was 1.2 and for 10-city TSPs was 1.3. These averages and the results shown in Figure 4 lead to the following conjectures.

Conjecture 1. Let the distances be integer values in $[1,21]$. As the number of cities increases, the number of TPSs with one optimal tour decreases.

Conjecture 2. Let the distances be integer values in $[1,21]$. As the number of cities increases, the probability increases that a random TPS has multiple optimal tours.

The wall time to find all optimal tours on 1000 10-city TSPs was about 2 minutes. The time on a digital computer is expected to be prohibitive for 1000 TSPs with 20 or more cities, but not for a quantum annealing computer with sufficient qubits and couplings between qubits. The anneal time to determine a minimum is negligible on a quantum machine; only time for input and output is significant.

\section{Summary}

The traveling salesman problem (TSP) has important real-world applications. When the number of cities is about 20 or more, exact solutions require a massive amount of time on digital computers. It is not known if quantum computers can efficiently solve NP-hard problems, like the TSP. Regardless of the answer, quantum computers are expected to have a significant advantage of finding optimal tours for TSPs quickly.

We anticipate that quantum annealing computers will find all optimal tours for a TSP. We have classified this output for 6, 8 and 10-city TSPs, which is the estimated number of cities that can be solved currently on a quantum annealing processor. Data from the study shows for large samples that the number of optimal tours for a TSP increases as the length of the optimal tour increases. This was true for $100 \%$ of the series for 6-city TSPs and 90\% for 8-city TSPs and 10-city TSPs.

There are no existing results about all optimal tours for a TSP. The data reported in this paper is the first to be published. It anticipates the quantum annealing results for small TSPs and opens the dialogue for conjecture about all optimal tours for TSPs with 20 or more cities.

\section{$5 \quad$ Future Work}

A next step is to input TSPs as formulated in $[11,19]$ into a D-Wave processor, verify the code, and validate the method for TSPs of various sizes and characteristics. Having done this, we are ready to randomly generate TSPs and analyze the patterns of outputs. All of this will necessitate solving the TSPs on a digital machine in order to confirm optimal tours.

There are significant questions to be explored with experimental data. Since the quantum annealing computer is analog in nature, to what extent can optimal tours be found? What is the largest integer $n$ for which a random TSP on $n$-cities can be solved on a current quantum annealing computer?

While waiting for permission to access a D-Wave quantum processor, small TSPs in the TSBLIB [17] can be studied to determine how many optional tours they have. This includes "burma14" which is symmetric, having 14 cities and an optimal tour whose length is 3323; and "br17" which is asymmetric, having 17 cities and an optimal tour whose length is 39. Can we successfully reduce the number of cities for these TSPs, solve the reduced TSP, and recover an optimal tour for the original problem [21]?

It has not escaped our attention that all of the TSPs in our study had an even number of cities. A TSP on $n$-cities when $n$ is a prime number has additional structure: If $\mathrm{T}$ is any tour, then $\mathrm{T}=\mathrm{T}^{1}, \mathrm{~T}^{2}, \ldots$, 
$\mathrm{T}^{\mathrm{n}-1}$ are tours and each member of the distance matrix is used exactly once to compute the lengths of these $n-1$ tours. By $\mathrm{T}^{\mathrm{i}}$ we mean the permutation $\mathrm{T}$ applied $i$ times. Also, if $\mathrm{T}$ is a tour for a TSP with an odd number $n$ of cities, then there are tours $\mathrm{T}=\mathrm{T}_{1}, \mathrm{~T}_{2}, \ldots, \mathrm{T}_{\mathrm{n}-1}$ which use each member of the distance matrix exactly once when computing their lengths. The tours $\mathrm{T}_{2}, \ldots, \mathrm{T}_{\mathrm{n}-1}$ have a special construction. Apparently, tours on an even number of cities do not have these properties. This theoretical hypothesis is open for investigation.

Acknowledgements. Data for this research was generated with Advanced Cyber Infrastructure computational resources provided by The Institute for CyberScience at The Pennsylvania State University. Professor Charles Helou sponsored use of these resources. A reviewer is thanked for suggestions that enhanced the paper. Also, I thank the Lord Jesus who has given me motivation and financial resources to investigate these topics and write this paper.

\section{References}

1. J. Bang, J. Ryu, C. Lee, S. Yoo, J. Lim, J. Lee, A quantum heuristic algorithm for the traveling salesman problem, J. Korean Phys. Soc. 61 (2012) 1944-1949. http://link.springer.com/article/10.3938/jkps.61.1944

2. G. Barach, H. Fort, Y. Mehlman, F. Zypman, Information in the traveling salesman problem, Applied Mathematics 3 (2012) 926-930. http://dx.doi.org/10.4236/am.2012.38138

3. Z. Bian, F. Chudak, R. Israel, B. Lackey, W. G. Macready, A. Roy, Discrete optimization using quantum annealing on sparse Ising models. Front. Phys. 2 Article 56 (2014) 10 pages. DOI:10.3389/fphy.2014.00056

4. H. Chen, X. Kong, B. Chong, G. Qin, X. Zhou, X. Peng, J. Du, Experimental demonstration of a quantum annealing algorithm for the traveling salesman problem in a nuclear-magnetic-resonance quantum simulator, Phys. Review A 83 (2011) 5 pages. DOI:10.1103/PhysRevA.83.032314

5. W. J. Cook, In Pursuit of the Traveling Salesman: Mathematics at the Limits of Computation. Princeton University Press, Princeton, NJ. 2012.

6. E. Dahl, Travelling Salesman Problem, D-Wave Systems Tutorial (Sep. 2013). Viewed Jan. 20, 2017 from http://web.archive.org/web/20130910030209/http://www.dwavesys.com/en/dev-tutorial-tsp.htm

7. G. Gutin, A. P. Punnen (editors), The Traveling Salesman Problem and Its Variations, Kluwer Academic Publishers, Dordrecht, The Netherlands, 2002.

8. J. Inoue, Infinite-range transverse field Ising models and quantum computation, Eur. Phys. J. Special Topics 224 (2015) 149-161. DOI:10.1140/epjst/e2015-02348-x

9. S. R. Kumar, T. S. Lamba, The travelling salesman cipher, Journal IETE 40 (1994) 151-154.

10. G. Laporte, A concise guide to the traveling salesman problem, J. Operational Res. Soc. 61 (2010) 35-40. DOI: $10.1057 /$ jors. 2009.76

11. A. Lucas, Ising formulations of many NP problems. Front. Phys. 2 Article 5 (2014) 15 pages. DOI:10.3389/fphy.2014.00005

12. R. Martoňák, G. E. Santoro, E. Tosatti, Quantum annealing of the traveling salesman problem, Phys. Rev. E 70 (2004) 5 pages. DOI:10.1103/PhysRevE.70.057701

13. C. C. McGeoch, C. Wang, Experimental evaluation of an adiabatic quantum system for combinatorial optimization, in Proceedings of the ACM International Conference on Computing Frontiers (2013 Proceedings), Article No. 23, ACM Press, New York, 2013. DOI:10.1145/2482767.2482797

14. H. R. Moser, The quantum mechanical solution of the traveling salesman problem, Phys. E 16 (2003) 280-285. DOI:10.1016/S1386-9477(02)00928-1

15. G. E. Santoro, E. Tosatti, Optimization using quantum mechanics: quantum annealing through adiabatic evolution, J. Phys. A 39 (2006) R393-R431. DOI:10.1088/0305-4470/39/36/R01

16. S. Suzuki, J. Inoue, B. K. Chakrabarti, Quantum Ising Phases and Transitions in Transverse Ising Models, 2nd edition, Lecture Notes in Physics 862, Springer, Berlin, 2013. DOI:10.1007/978-3-642-33039-1

17. TSPLIB is a library of sample instances for the TSP available at http://comopt.ifi.uni-heidelberg.de/software/TSPLIB95/tsp95.pdf

18. E. Tsukerman, Ping-pong and the traveling salesman problem, Experimental Mathematics, 25 (2016) 1-7. DOI: $10.1080 / 10586458.2014 .1002140$ 
19. R. H. Warren, Adapting the traveling salesman problem to an adiabatic quantum computer, Quantum Inf. Process. 12 (2013) 1781-1785. DOI:10.1007/s11128-012-0490-8

20. R. H. Warren, Special Cases of the Traveling Salesman Problem. Applied Mathematics and Computation 60 (1994) 171-177. DOI: 10.1016/0096-3003(94)90103-1

21. R. H. Warren, Optimal Arcs for the Traveling Salesman Problem, Applied Mathematics Letters 5 (1992) 13-14. DOI: 10.1016/0893-9659(92)90028-8 